\title{
Trombose venosa profunda num membro superior em mulher a fazer anticoncepcional oral e com trombofilia hereditária - Factor V Leiden
}

Helena Oliveira Maia*

\section{RESUMO}

Introdução: A trombose venosa profunda (TVP) dos membros superiores representa cerca de $10 \%$ dos casos de TVP. Factores genéticos e adquiridos contribuem para a trombose venosa. O factor $\vee$ Leiden é a trombofilia hereditária mais comum. $\mathrm{O}$ uso de anticoncepcionais orais é também um factor de risco reconhecido de TVP. Neste artigo é apresentado o caso de uma mulher a fazer anticoncepcional oral combinado, heterozigótica para o factor $V$ Leiden, que desenvolve uma TVP do membro superior.

Descrição do caso: Mulher de 34 anos, caucasiana, recorre ao serviço de urgência por dor e edema do membro superior direito, com menos de 24 horas de evolução. Nega dispneia ou dor torácica. Sem história recente de trauma. Não apresenta problemas médicos crónicos. Iniciou anticoncepcional oral combinado há dois meses. História médica familiar negativa para eventos tromboembólicos. O ecodoppler venoso identificou trombose da porção terminal da veia subclávia direita, com cerca de $4 \mathrm{~cm}$ de extensão. Iniciou anticoagulação com enoxaparina $1 \mathrm{mg} / \mathrm{kg} / \mathrm{dia}$, tendo completado seis meses de anticoagulação com acenocumarol, com o valor alvo de INR 2.0-3.0. O anticoncepcional oral combinado foi interrompido. O rastreio de trombofilias, realizado após interrupção da anticoagulação, revelou heterozigotia para o factor $\vee$ Leiden.

Comentário: O factor $\vee$ Leiden contribui para o risco de tromboembolismo venoso geralmente em combinação com outro factor de risco adicional, por exemplo, o uso de contraceptivos orais. Os indivíduos com esta mutação devem ser aconselhados a reduzir ou eliminar outros factores de risco que contribuem para o desenvolvimento do tromboembolismo venoso.

Palavras-chave: Factor V Leiden; Trombose Venosa; Contracepção Oral Combinada.

\section{INTRODUÇÃO}

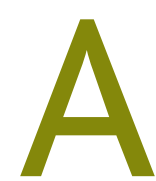

patogénese da trombose venosa é complexa e multifactorial e resulta da interacção entre factores hereditários e adquiridos. ${ }^{1-3}$ Aproximadamente $10 \%$ dos casos de trombose venosa profunda (TVP) ocorrem nos membros superiores. A incidência tem vindo a aumentar ao longo do tempo, resultado de um maior número de casos de trombose venosa secundária ao uso de dispositivos

* Médica Interna de Formação Específica de Medicina Geral e Familiar USF Porta do Sol - Unidade Local de Saúde de Matosinhos médicos, nomeadamente cateteres venosos centrais. ${ }^{4-5}$ Cerca de 20 a $30 \%$ dos casos de TVP nos membros superiores são primários, sem aparente causa precipitante ou na presença de variantes anatómicas; os restantes casos são secundários e estão associados ao uso de cateteres venosos centrais, à presença de neoplasia, a cirurgia recente ou trauma. ${ }^{4-6}$

O factor V Leiden é o factor de risco tromboembólico venoso hereditário mais comum..$^{2-3} \mathrm{~A}$ sua prevalência é mais elevada na população europeia e varia entre $2 \%$ e $7 \%$, sendo menos prevalente entre os africanos e asiáticos. ${ }^{7-8}$ Em heterozigotia, o factorV Leiden está pre- 
sente em cerca de $5 \%$ dos indivíduos de raça caucasiana, aumentando o risco de trombose venosa entre 5 a 10 vezes. $^{8}$ No entanto, apesar do aumento de risco relativo, o risco absoluto é baixo., ${ }^{3,-8}$ Os casos de tromboembolismo venoso em mulheres em idade reprodutiva são pouco frequentes, menos de 1 por 10.000 pessoas-ano. ${ }^{7}$ Os indivíduos homozigóticos para o factor $\mathrm{V}$ Leiden apresentam um risco de trombose venosa entre 20 a 50 vezes superior. ${ }^{3,8}$

Geralmente é a combinação entre a presença de factorV Leiden e outro factor de risco adicional que condiciona o fenómeno trombótico. ${ }^{1-3,8}$ Esses factores de risco incluem: idade, cirurgia, obesidade, imobilização prolongada, hospitalização, uso de contraceptivos orais, gravidez, neoplasia, entre outros. ${ }^{1-3,8}$

A contracepção oral combinada é um factor de risco de tromboembolismo venoso reconhecido, aumentando entre 3 a 6 vezes o risco trombótico. ${ }^{7-8} \mathrm{Em} \mathrm{mu-}$ lheres com factor $V$ Leiden esse risco é acrescido. ${ }^{7}$

\section{DESCRIÇÃO DO CASO CLÍNICO}

Mulher de 34 anos, caucasiana, autónoma, operadora de caixa de supermercado. Sem antecedentes médicos ou cirúrgicos relevantes. Iniciou anticoncepcional oral combinado há cerca de dois meses (etinilestradiol/gestodeno-0,02/0,075mg), sem outras medicações crónicas. Sem hábitos tabágicos ou etílicos. Pratica exercício físico regularmente (IMC: $20 \mathrm{~kg} / \mathrm{m}^{2}$ ). História ginecológica: 0G0P. Sem antecedentes familiares relevantes.

Recorreu ao Serviço de Urgência (SU) por alteração da coloração do braço direito e "sensação de inchaço" (sic), acompanhado de dor e dormência, com extensão ao antebraço homolateral, quadro com menos de 24 horas de evolução. Nega dispneia ou dor torácica. Nega história de traumatismo recente. História familiar negativa para eventos tromboembólicos. Ao exame objectivo: sem sinais de dificuldade respiratória. Temperatura axilar $-36,7^{\circ} \mathrm{C}$, tensão arterial $-113 / 70 \mathrm{mmHg}$, frequência cardíaca - 63 batimentos por minuto. Auscultação cardíaca e pulmonar sem alterações. Sem turgescência venosa jugular. $\mathrm{O}$ membro superior direito (MSD) apresentava pele marmoreada, edema discreto, quente e ligeira dor à palpação. Pulso arterial presente e simétrico em ambos os membros superiores. Força muscular mantida. Sinais de circulação venosa superficial colateral no ombro direito e face anterior do tó- rax, mais evidente à direita.

Foi realizado estudo complementar: hemoglobina 13,4g/dL; plaquetas - 239000/L; leucócitos - 9300/L; creatinina $-0,7 \mathrm{mg} / \mathrm{dL}$; glicose $-78 \mathrm{mg} / \mathrm{dL}$; proteína $\mathrm{C}$ reactiva $-2,9 \mathrm{mg} / \mathrm{dL}$; tempo de tromboplastina activada-24,3 s; tempo de protrombina-10,4 s e D-dímeros $-3319,9 \mathrm{ng} / \mathrm{mL}$. Este último parâmetro foi o único que não se encontrava dentro dos valores de referência, apresentando uma elevação (normal $<500 \mathrm{ng} / \mathrm{mL}$ ).

Por suspeita de trombose venosa profunda do MSD realizou ecodoppler que confirmou trombose da porção terminal da veia subclávia direita, com cerca de $4 \mathrm{~cm}$ de extensão, imediatamente antes da união com a veia jugular interna homolateral.

A doente iniciou anticoagulação ainda no SU, com enoxaparina $1 \mathrm{mg} / \mathrm{kg} / \mathrm{dia}$, tendo posteriormente, já no internamento, começado anticoagulação oral com acenocumarol. $\mathrm{O}$ anticoncepcional oral foi interrompido. O internamento decorreu sem intercorrências. Realizou hipocoagulação durante seis meses, tendo como valor alvo de INR 2.0-3.0. O novo método anticoncepcional passou a ser o preservativo masculino.

Após parar hipocoagulação, a doente realizou em ambulatório, na consulta de hematologia, estudo de trombofilias, que incluiu o doseamento de fibrinogénio, anticoagulante lúpico, anticorpos antifosfolípidos, antitrombina III, proteína S, proteína C, homocisteína e pesquisa de mutação do factor $\mathrm{V}$ e da protrombina. $\mathrm{O}$ estudo revelou heterozigotia para o factor $V$ Leiden.

\section{COMENTÁRIO}

As manifestações clínicas mais comuns de TVP dos membros superiores são a sensação de peso, desconforto, dor, parestesias e edema do membro afectado. Ao exame objectivo pode observar-se edema e alteração da coloração (rubor ou cianose) da extremidade em causa e circulação venosa colateral no ombro ou antebraçO. $^{4-5}$

A avaliação clínica apresenta baixa especificidade (30-64\%). Estudos imagiológicos são necessários perante a suspeita de TVP dos MS. ${ }^{4}$

No caso clínico exposto, a doente apresentava um quadro clínico sugestivo de trombose venosa profunda do MSD. Realizou ecodoppler venoso do MSD, que confirmou trombose da porção terminal da veia subclávia direita. 
A investigação dos factores de risco para este evento trombótico venoso revelou uma história pessoal e familiar negativa para eventos tromboembólicos venosos. Ausência de eventos traumáticos recentes ou cirurgias prévias. O estudo trombofílico realizado posteriormente identificou a presença de heterozigotia para o factorV Leiden. Para além da presença deste último factor de risco, a doente relata o início recente, cerca de dois meses antes, de anticoncepcional oral combinado.

O estudo de Martinelli et al revelou que a heterozigotia para o factor V Leiden aumenta o risco de TVP do MS em cerca de seis vezes e que este é 14 vezes superior no caso da combinação factorVLeiden e uso de anticoncepcional oral combinado, comparativamente à ausência desses factores de risco. ${ }^{6}$

As indicações da duração do tempo de anticoagulação são ainda incertas na TVP dos membros superiores. ${ }^{4}$ A duração do tratamento é baseada nos factores de risco que condicionaram o evento trombótico. ${ }^{3}$

$\mathrm{O}$ risco de recorrência de TVP dos MS é inferior ao observado nos membros inferiores, não existindo indicação para anticoagulação prolongada. ${ }^{4,6}$

$\mathrm{O}$ factor V Leiden não aumenta necessariamente o risco de recorrência de eventos trombóticos em indivíduos com factor de risco transitório. Assim, indivíduos heterozigóticos para esta mutação, com um único episódio de TVP ou tromboembolismo pulmonar, não necessitam habitualmente de realizar anticoagulação para toda a vida, a não ser que existam factores de risco adicionais que o possam justificar. ${ }^{3}$

$\mathrm{O}$ factor $\mathrm{V}$ Leiden é um de vários factores de risco para TVP. Esse risco será maior quanto mais factores de risco existirem. Por vezes, o efeito de múltiplos factores de risco é superior à adição do seu efeito individual. ${ }^{3,8}$ Assim sendo, os indivíduos que apresentem esta mutação deverão ser aconselhados a reduzir ou a eliminar outros factores de risco trombóticos. Alguns desses factores, como a genética e a idade, não são modificáveis; no entanto, outros factores podem ser controlados, nomeadamente: redução do peso (no caso de excesso de peso ou obesidade), evitar longos períodos de imobilização, permanecer activo e realizar actividade física regular, evitar o tabagismo, realizar tromboprofilaxia nas situações indicadas. $O$ doente deve também ser educado para reconhecer os sintomas e sinais de TVP ou tromboembolismo pulmonar e procurar ajuda médica atempadamente.,

No caso do sexo feminino, um aspecto importante a abordar é a contracepção. A Organização Mundial da Saúde (OMS) recomenda que mulheres com mutação trombogénica reconhecida não deverão utilizar anticoncepcionais orais combinados (Categoria 4 da OMS), mas podem usar métodos anticoncepcionais apenas com progestativo (Categoria 2 da OMS), dispositivos intrauterinos de cobre e com progestativo (Categoria 1 e 2, respectivamente, da OMS) e métodos de barreira (Categoria $1 \mathrm{da}$ OMS). ${ }^{9}$ Segundo a OMS, o rastreio por rotina de mutações trombogénicas não é adequado, tendo em conta a raridade da condição e o elevado custo do rastreio.

Relativamente ao rastreio de factor V Leiden em familiares assintomáticos de indivíduos afectados e com história de trombose venosa deverá ser feito numa base individual, tendo em conta os seus factores de risco adicionais, não sendo recomendado por rotina. ${ }^{3-8}$

O presente caso clínico retrata a componente multifactorial da trombose venosa, em que se associa um factor de risco hereditário (FactorV Leiden) e um factor de risco adquirido (uso de anticoncepcional oral combinado).

\section{REFERÊNCIAS BIBLIOGRÁFICAS}

1. Konkle BA. Bleeding and thrombosis. In Longo DL, Fauci AS, Kasper DL, Hauser SL, Jameson JL, Loscalzo J, editors. Harrison's - Principles of internal medicine. 17th ed. Washington: McGraw-Hill; 2008. p. 363-9. ISBN 9780071466332

2. Rosendaal FR, Buller HR. Venous thrombosis. In Longo DL, Fauci AS, Kasper DL, Hauser SL, Jameson JL, Loscalzo J, editors. Harrison's - Principles of internal medicine. 17th ed. Washington: McGraw-Hill; 2008. p. 731-5. ISBN 9780071466332

3. Shaheen K, Alraies MC, Alraiyes AH, Christie R. Factor V Leiden: how great is the risk of venous thromboembolism? Cleve Clin J Med. 2012;79(4):265-72.

4. Grant JD, Stevens SM, Woller SC, Lee EW, Kee ST, Liu DM, et al. Diagnosis and management of upper extremity deep-vein thrombosis in adults. Thromb Haemost. 2012;108(6):1097-108.

5. Engelberger RP, Kucher N. Management of deep vein thrombosis of the upper extremity. Circulation. 2012;126(6):768-73.

6. Martinelli I, Battaglioli T, Bucciarelli P, Passamonti SM, Mannucci PM. Risk factors and recurrence rate of primary deep vein thrombosis of upper extremities. Circulation. 2004;110(5):566-70.

7. Mohlajee AP, Curtis KM, Martins SL, Peterson HB. Does use of hormonal contraceptives among women with thrombogenic mutations increase their risk of venous thromboembolism? A systematic review. Contraception. 2006;73(2):166-78.

8. Ornstein DL, Cushman M. Cardiology patient page: factor $V$ Leiden. 
Circulation. 2003;107(15):e94-7.

9. World Health Organization. Medical eligibility criteria for contraceptive use. 4th ed. Geneva:WHO; 2010. ISBN 9789241563888

\section{CONFLITO DE INTERESSES}

A autora declara não ter conflitos de interesses.

\section{ENDEREÇO PARA CORRESPONDÊNCIA}

Helena Oliveira Maia

E-mail: helenaisabelmaia@gmail.com

Recebido em 09-12-2014

Aceite para publicação em 22-03-2015

\section{ABSTRACT}

\section{UPPER-EXTREMITY DEEP VENOUS THROMBOSIS IN A WOMAN ON HORMONAL ORAL CONTRACEPTION WITH AN INHERITED THROMBOPHILIA - FACTOR V LEIDEN}

Introduction: Upper extremity deep vein thrombosis (DVT) represents $10 \%$ of all cases of DVT. Genetic and acquired factors contribute to DVT. Factor $\mathrm{V}$ Leiden is the most common inherited thrombophilia. The use of oral contraceptives is also a recognized risk factor for DVT. The case of a female patient receiving a combined oral contraceptive who developed a upper-extremity deep venous thrombosis is presented.

Case report: A 34 year-old Caucasian female presented to the emergency department with pain and swelling of the right arm. These symptoms began suddenly less than 24 hours earlier. She denied shortness of breath or thoracic pain. She had not experienced recent trauma. The patient had no chronic medical problems. She had been taking a combined oral contraceptive for two months. Her family medical history was negative for thromboembolic events. Doppler ultrasonography showed a venous thrombosis of terminal portion of right subclavian vein with $4 \mathrm{~cm}$ of extension. Anticoagulation therapy was started in the emergency department with enoxaparin $1 \mathrm{mg} / \mathrm{kg} /$ day. This was followed by 6 months of oral anticoagulation therapy with acenocoumarol with a target INR of 2.0-3.0. The combined oral contraceptive was stopped. Thrombophilia screening was performed after completing anticoagulation. The patient was found to be heterozygous for factor $V$ Leiden.

Discussion: Factor $V$ Leiden contributes to the risk of venous thromboembolism often in combination with an additional risk factor, such as oral contraceptive use. Patients should be counseled about reducing or eliminating other risk factors that may contribute to the development of venous thromboembolism.

Keywords: Factor V Leiden; Venous Thrombosis; Oral Hormonal Contraception. 